\title{
ACCVD Growth, Raman and Photoluminescence Spectroscopy of Isotopically Modified Single-Walled Carbon Nanotubes
}

\author{
Shigeo Maruyama and Yuhei Miyauchi \\ Department of Mechanical Engineering, The University of Tokyo \\ 7-3-1 Hongo, Bunkyo-ku, Tokyo 113-8656, Japan
}

\begin{abstract}
Using alcohol catalytic CVD (ACCVD) technique optimized for the efficient production of SWNTs from very small amount of ethanol, SWNTs consisting of carbon-13 isotope $\left(\mathrm{SW}^{13} \mathrm{CNTs}\right.$ ) were synthesized. Raman scatterings from $\mathrm{SW}^{13} \mathrm{CNTs}$ show no change from $\mathrm{SW}^{12} \mathrm{CNTs}$ in spectrum shape except for the Raman shift frequency down-shifted as much as square-root of mass ratio 12/13. On the other hand, SWNTs with mixed 12/13 isotopes from ${ }^{13} \mathrm{CH}_{3}-\mathrm{CH}_{2}-\mathrm{OH}$ and $\mathrm{CH}_{3}{ }^{-13} \mathrm{CH}_{2}-\mathrm{OH}$ show different amount of down-shift in Raman spectra, suggesting that smaller amount of site-1 carbon atom (next to $\mathrm{OH}$ ) is incorporated into SWNTs. Based on this result, the initial decomposition reaction of ethanol on metal catalyst is discussed. Furthermore, near infrared luminescence of $\mathrm{D}_{2} \mathrm{O}$-surfactant dispersions of both $\mathrm{SW}^{13} \mathrm{CNTs}$ and $\mathrm{SW}^{12} \mathrm{CNT}$ were mapped. By comparing these maps, phonon sidebands of excitonic excitation were clearly identified.
\end{abstract}

Keywords: Single-Walled Carbon Nanotubes, Alcohol CVD, Raman, Photoluminescence.

PACS: 78.67.Ch, 81.07.De

\section{INTRODUCTION}

For the production technique of single-walled carbon nanotubes (SWNTs), various CVD approaches are being developed in stead of laser-furnace and arc-discharge methods. At present, CVD approaches using the high-pressure CO (HiPco) [1] is dominant for the mass production of SWNTs. Recently, we have proposed the use of alcohol for the carbon feedstock [2,3] for the generation of high-purity SWNTs at low temperatures. Furthermore, it was demonstrated that vertically aligned high quality SWNTs mat could be synthesized directly on silicon and quartz substrates [4]. This versatile nature of this technique made it possible to use rather expensive isotopically modified ethanol for the production of SWNTs. In this report, ACCVD method is modified for the efficient production of SWNTs from very small amount of ethanol with the similar technique used for the SWNTs generation from fullerene [5]. The SWNTs with various amount of ${ }^{13} \mathrm{C}$ abundance were generated by ACCVD technique from isotopically modified ethanol: ${ }^{13} \mathrm{CH}_{3^{-}}{ }^{13} \mathrm{CH}_{2}-\mathrm{OH},{ }^{13} \mathrm{CH}_{3}-\mathrm{CH}_{2}-\mathrm{OH}$, and $\mathrm{CH}_{3}-$ ${ }^{13} \mathrm{CH}_{2}-\mathrm{OH}$, in addition to the normal ethanol. The resonant Raman scattering of those SWNTs shows a simple shift of Raman scatting frequency for ${ }^{13} \mathrm{C}$ SWNTs. On the other hand, SWNTs with mixed 12/13 isotopes from ${ }^{13} \mathrm{CH}_{3}-\mathrm{CH}_{2}-\mathrm{OH}$ and $\mathrm{CH}_{3}{ }^{-13} \mathrm{CH}_{2}-$ 
$\mathrm{OH}$ show different amount of down-shift in Raman spectra, suggesting that smaller amount of site- 1 carbon atom (next to $\mathrm{OH}$ ) is incorporated into SWNTs. Based on this result, the initial decomposition reaction of ethanol on metal catalyst is discussed.

Photoluminescence (PL) has been intensively studied for the characterization of SWNTs [6, 7]. By plotting PL emission intensities as a function of emission and excitation photon energy, each peak in the PL-map is assigned to each chirality (n, m) [7]. As far as semiconductor SWNTs, PL-map is the most promising approaches for a quick determination of the structure distribution on a bulk SWNT sample [8], Hence, photoluminescence spectroscopy is a powerful tool not only for investigations of electronic properties of SWNTs but challenges to the $(n, m)$-controlled synthesis of SWNTs. However, in a PL map, we can find some PL peaks whose origins have not been elucidated other than bright PL peaks already assigned to particular nanotube (n, m) $[7,8]$. Since these unassigned features may overlap with other PL peaks if a measured sample is an assemblage of various (n, $m$ ) structures, it is very important to understand the origins of all the features in a PL map for the accurate measurement of relative PL intensities of each $(n, m)$ nanotube. Phonon assisted excitonic recombination [9] or phonon sideband due to strong phonon-exciton interaction [10] are proposed for some of extra excitation features. By using isotopically modified SWNTs with different phonon energies, PL peaks originates from exciton-phonon (exciton-phonon) interactions can be clearly distinguished from PL peaks without exciton-phonon interaction.

\section{EXPERIMETAL PROCEDURES}

\section{Alcohol CVD Growth of Isotopically Modified SWNTs}

We synthesized $\mathrm{SW}^{13} \mathrm{CNT}$ from 0.5 gram of isotope-modified ethanol by alcohol catalytic chemical vapor deposition (ACCVD) method [2, 3] optimized for the efficient production of SWNTs from very small amount of ethanol, which is similar to the technique used for the SWNT synthesis from fullerene [5]. The detailed preparation of metal supporting zeolite powder was described in our previous reports $[2,3]$. We prepared a catalytic powder by impregnating iron acetate $\left(\mathrm{CH}_{3} \mathrm{CO}_{2}\right)_{2} \mathrm{Fe}$ and cobalt acetate $\left(\mathrm{CH}_{3} \mathrm{CO}_{2}\right)_{2} \mathrm{Co}-4 \mathrm{H}_{2} \mathrm{O}$ onto USY-zeolite powder (HSZ-390HUA over $99 \% \mathrm{SiO}_{2}$ ). The weight concentration of $\mathrm{Fe}$ and $\mathrm{Co}$ was chosen to be $2.5 \mathrm{wt} \%$ each over the catalytic powder. A slightly modified apparatus from our standard ACCVD technique was employed as shown in Fig. 1. The catalyst was placed on a quartz boat and the boat was set in the center of a quartz tube (i.d. $=26 \mathrm{~mm}$, length $=1 \mathrm{~m}$ ). Small amount of ethanol was placed in an end of a smaller test-tube guiding the carbon source flow to the catalyst. One end of the quartz tube was connected to a rotary pump by two different paths, one $25 \mathrm{~mm}$ and the other $6 \mathrm{~mm}$ diameter tubes to select the pumping efficiency. The central $30 \mathrm{~cm}$ of the quartz tube was surrounded with an electric furnace. While the furnace was heated up from room temperature, about 200 sccm of Ar was flowed so that the inside of the quartz tube was maintained at $1 \mathrm{~atm}$. After the electric furnace reached desired temperature, $800{ }^{\circ} \mathrm{C}$, Ar flow was stopped and the larger evacuation path was opened to bring the inside of the quartz tube 
vacuum. Subsequently, ethanol vapor was injected to catalyst for 5 min from the end of the smaller test-tube ethanol reservoir. The synthesized SWNTs were characterized by micro Raman scattering measurements using CHROMEX 501is and ANDOR DV401-FI for the spectrometer and CCD system, respectively, with an optical system of SEKI TECHNOTRON STR250.

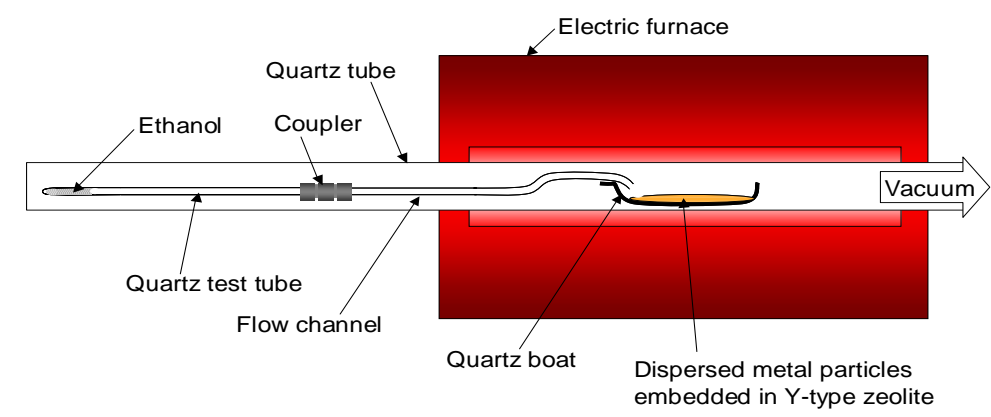

FIGURE 1. Schematic diagram of alcohol CVD apparatus for efficient generation of SWNTs

\section{Dispersion and Photoluminescence Spectroscopy}

In order to measure PL spectra from individual SWNTs in surfactant suspension, 'as-grown' materials were dispersed in $\mathrm{D}_{2} \mathrm{O}$ with $0.5 \mathrm{wt} \%$ sodium dodecylbenzene sulfonate (NaDDBS) by heavy sonication with an ultrasonic processor (Hielscher GmbH, UP-400S with H3/Micro Tip 3) for $1 \mathrm{~h}$ at a power flux level of $460 \mathrm{~W} / \mathrm{cm}^{2}$. These suspensions were then centrifuged (Hitachi Koki himac CS120GX with S100AT6 angle rotor) for $1 \mathrm{~h}$ at $386000 \mathrm{~g}$ and the supernatants, rich in isolated SWNTs, were used in the PL measurements.

Near infrared emission from the samples were recorded while the excitation wavelength was scanned from VIS to NIR range. The measured spectral data were corrected for wavelength-dependent variations in excitation intensity and detection sensitivity. The excitation and emission spectral slit widths were $10 \mathrm{~nm}$ (15 30 meV for excitation and $\sim 10 \mathrm{meV}$ for emission in the measuring range), and scan steps were $5 \mathrm{~nm}$ on both axes. In addition to PL maps of wide energy range of emission, we scanned PLE spectra of $(7,5)$ nanotubes (emission at $1026.5 \mathrm{~nm}(1.208 \mathrm{eV})$ ) with narrower excitation spectral slit width (5 nm : 8 15 meV in the measuring range) and scan steps $(2 \mathrm{~nm})$ to obtain spectra with higher resolution. The photoluminescence spectra were measured with a HORIBA SPEX Fluorolog-3-11 spectrofluorometer with a liquid-nitrogen-cooled InGaAs near IR detector.

\section{RAMAN SPECTRA AND GROWTH MECHANISM}

\section{Raman Spectra of Isotopically Modified SWNTs}

In addition to normal ethanol, 3 isotopically modified ethanol were employed for the production of isotopically modified SWNTs. Three isotopically modified ethanol were ${ }^{13} \mathrm{CH}_{3}-{ }^{13} \mathrm{CH}_{2}-\mathrm{OH}\left(1,2-{ }^{13} \mathrm{C}_{2}, 99 \%\right),{ }^{13} \mathrm{CH}_{3}-\mathrm{CH}_{2}-\mathrm{OH}\left(2-{ }^{13} \mathrm{C}, 99 \%\right), \mathrm{CH}_{3^{-}}{ }^{13} \mathrm{CH}_{2}-\mathrm{OH}$ (1- 
${ }^{13} \mathrm{C}$, 98\%), supplied from Cambridge Isotope Laboratories, Inc. The carbon atoms in normal ethanol $\mathrm{CH}_{3}-\mathrm{CH}_{2}-\mathrm{OH}$ are composed of isotope mixtures of natural abundance, ie. $98.892 \%{ }^{12} \mathrm{C}$ and $1.108 \%{ }^{13} \mathrm{C}$. Hence, by using normal ethanol, generated SWNTs should be composed of carbon atoms with the same abundance ratio. On the other hand, by using ethanol $\left({ }^{13} \mathrm{CH}_{3}{ }^{13}{ }^{13} \mathrm{CH}_{2}-\mathrm{OH}\right)$, SWNTs principally made of ${ }^{13} \mathrm{C}$ can be generated. By using other 2 isotopically labeled ethanol $\left({ }^{13} \mathrm{CH}_{3}-\mathrm{CH}_{2}-\mathrm{OH}\right.$ or $\mathrm{CH}_{3}-$ $\left.{ }^{13} \mathrm{CH}_{2}-\mathrm{OH}\right)$, the abundance of ${ }^{13} \mathrm{C}$ isotopes in generated SWNTs should depend on the reaction process.
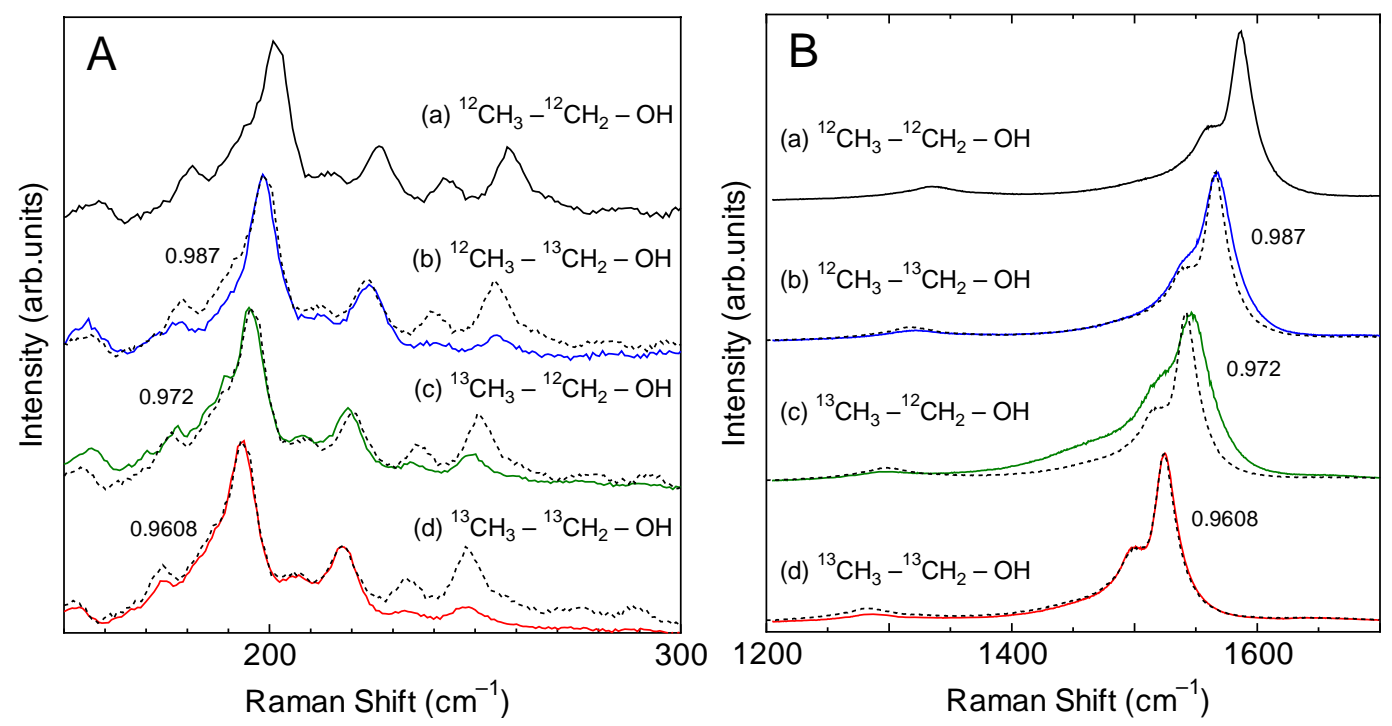

FIGURE 2. Comparison of RBM (A) and G-band (B) of SWNTs generated from various isotopically modified ethanol. (a): SWNTs from ${ }^{12} \mathrm{CH}_{3^{-}}{ }^{12} \mathrm{CH}_{2}-\mathrm{OH}$. (b): SWNTs from ${ }^{12} \mathrm{CH}_{3^{-}}{ }^{13} \mathrm{CH}_{2}-\mathrm{OH}$. (c): SWNTs from ${ }^{13} \mathrm{CH}_{3}{ }^{-12} \mathrm{CH}_{2}$-OH. (d): ${ }^{13} \mathrm{CH}_{3^{-}}{ }^{13} \mathrm{CH}_{2}-\mathrm{OH}$. Dotted lines are shifted spectra of ${ }^{12} \mathrm{C}$ SWNTs by multiplying the following factor to the frequency: (b) 0.987, (c) 0.972, (d) $\sqrt{12 / 13}$.

Fig. 2 compares Raman scattering for 4 kinds of SWNTs from isotopically modified ethanol excited with $488 \mathrm{~nm}$ laser spectra. Spread in G-band peak at 1590 $\mathrm{cm}^{-1}$ for normal SWNTs and very small D-band signal at $1350 \mathrm{~cm}^{-1}$ suggest that high quality SWNTs were generated by these experiments from tiny amount of ethanol. The strong radial breathing mode (RBM) peaks at around $150-300 \mathrm{~cm}^{-1}$ onfirms this observation. By multiplying the mass-ratio factor $\sqrt{12 / 13}=0.9608$ to frequency of $\mathrm{SW}^{12} \mathrm{CNT}$ (Fig. 2(a)) spectrum, SW ${ }^{13} \mathrm{CNTs}$ (Fig. 2(d)) spectrum is almost completely reproduced, where dotted line is the shifted spectrum from $\mathrm{SW}^{12} \mathrm{CNT}$.

\section{The Decomposition of Ethanol on Catalyst}

The interpretation of 2 mixed isotope cases in Figs. 2 (b, c) is quite important. In addition to slight broadening of spectra, the different shifts of the frequency was observed for 2 mixed cases. The difference in the vibrational frequency between (b) from ${ }^{12} \mathrm{CH}_{3^{-}}{ }^{13} \mathrm{CH}_{2}-\mathrm{OH}$ and (c) from ${ }^{13} \mathrm{CH}_{3^{-}}{ }^{12} \mathrm{CH}_{2}-\mathrm{OH}$ means that two carbon atoms in an ethanol molecule are not equally used for the SWNT formation. Apparently, the 
carbon atom at site 2, further from $\mathrm{OH}$, is more likely to be incorporated into an SWNT. The scaling factor on frequency of the dotted lines for (b) and (c) were 0.972 and 0.987, respectively. Assuming that this factor is the frequency change due to the average mass of carbon atoms, the factor should equal to $\sqrt{12 / m_{\text {ave }}}$. Then, the average mass for (b) and (c) are $12.32 \mathrm{amu}$ (Site 1: Site 2 = 32:68) and $12.70 \mathrm{amu}$ (Site 1: Site $2=30: 70$ ), respectively. Both (b), (c) results conclude that only about $30 \%$ carbon atoms in an SWNT are from the site 1 carbon atom. This result gives an important key for the analysis of the reaction mechanism of nanotube formation. On the metal catalyst surface, ethanol is expected to decompose. After losing hydrogen atoms, we believe that the oxygen atom has an important role of cleaning the carbon atoms with dangling bonds [2].

Let's assume that the ratio of original site 1 carbon atom in an SWNT is $\alpha$, and that of site 2 is $(1-\alpha)$. If we further assume that the probability of complete break of C-O bond is $P_{\text {free, }}$ and the free oxygen atom can randomly find its partner to become gas phase CO. Then, the incorporation of site 1 carbon atom can be possible by the C-O breaking and the choice of originally site 2 carbon atom by this free oxygen atom. Hence the probability is described as $\alpha=P_{\text {free }} \times(1-\alpha)$. With $\alpha=30 \%, P_{\text {free }}$ can be calculated as $45 \%$. So, we can conclude that about half of $\mathrm{C}-\mathrm{O}$ bond of an original ethanol is completely broken in the typical ACCVD process.

\section{PHOTOLUMINESCENCE FROM ${ }^{13}$ C NANOTUBES}

Figure 3(a,b) shows PL maps for $\mathrm{SW}^{13} \mathrm{CNTs}$ and normal SWNTs and Fig. 3c compares PLE spectra corresponding to $(7,5)$ nanotubes (emission at $1.208 \mathrm{eV}$ ) for both $\mathrm{SW}^{13} \mathrm{CNTs}$ and $\mathrm{SW}^{12} \mathrm{CNTs}$. Peak positions of major PL peaks of $\mathrm{SW}^{13} \mathrm{CNT}$ sample were in good agreement with those of normal SWNT sample, indicating electronic properties and average environment around nanotubes are almost equivalent in each sample. Hence, only phonon energies are the principal difference between $\mathrm{SW}^{13} \mathrm{CNTs}$ and normal SWNTs. Small PL peaks around the main peaks were observed as shown in Fig.3. For example, we find three small peaks above and below the main PL peaks corresponding to $E_{11}\left(1.208 \mathrm{eV}\right.$, out of measuring range) and $\mathrm{E}_{22}$ $(1.923 \mathrm{eV})$ transition energies of $(7,5)$ nanotubes in Fig. 3. Since the emission energies of these peaks were almost identical with the emission energy of the main peak at $E_{22}$ excitation energy of $(7,5)$ nanotubes, these peaks are also attributed to photon emission of $(7,5)$ nanotubes. In this paper, we focus on these unassigned PL peaks of $(7,5)$ nanotubes. Hereafter, we refer to these unassigned peaks as peak A, B and C as shown in Fig. 3.

In the case of peak A and C, energy differences from the main peaks were clearly changed depending on whether the sample is $\mathrm{SW}^{13} \mathrm{CNTs}$ or $\mathrm{SW}^{12} \mathrm{CNTs}$, while positions of the main peaks were almost identical. If a certain PL peak is a phonon sideband corresponding to the main PL peak, the amount of the change of the energy difference between the main peak and its sideband should be consistent with the value estimated from the difference of phonon energies confirmed by Raman spectroscopy shown in Fig.2. Isotope shift for peak $A$ and $C$ confirmed that these peaks are phonon side-band from $E_{11}$ and $E_{22}$, respectively [11]. On the other hand, we observed almost 
no peak shift of peak B as shown in Fig.3c, indicating that peak B is due to 'pure electronic' transition without electron-phonon interaction. Further study shows that this peak is due to transverse excitation of nanotubes [12].
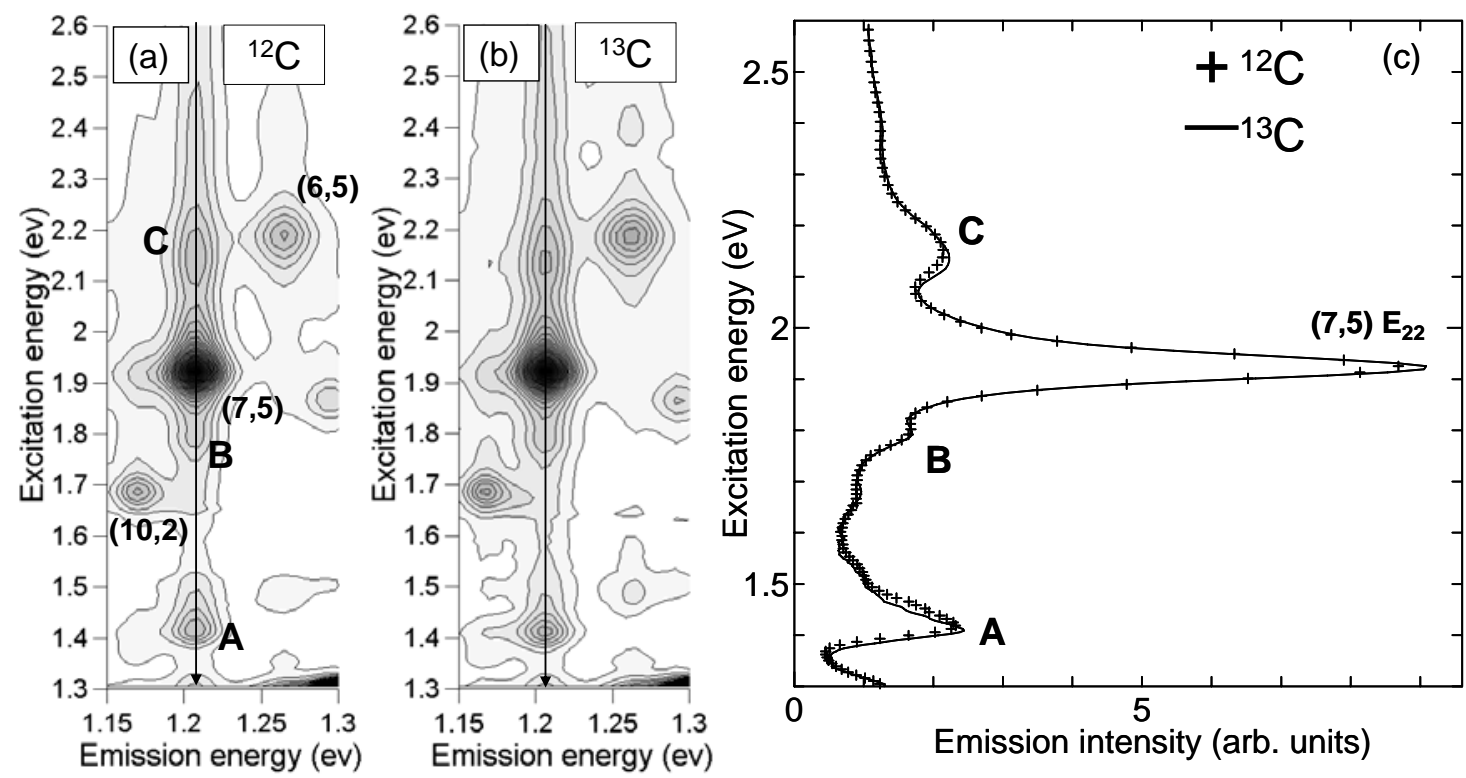

FIGURE 3. PL maps of (a) normal SWNTs and (b) $\mathrm{SW}^{13} \mathrm{CNTs}$ dispersed in surfactant suspension. (c) : Comparison of PLE spectra of SW ${ }^{13} \mathrm{CNTs}$ and normal SWNTs at the emission energy of $1.208 \mathrm{eV}$ (corresponding to E11 energy of $(7,5)$ nanotubes).

\section{REFERENCES}

1. P. Nikolaev, M.J. Bronikowski, R.K. Bradley, F. Rohmund, D.T. Colbert, K.A. Smith, R.E. Smalley, Chem. Phys. Lett., 313, 91-97 (1999).

2. S. Maruyama, R. Kojima, Y. Miyauchi, S. Chiashi, M. Kohno, Chem. Phys. Lett. 360, 229 (2002).

3. Y. Murakami, Y. Miyauchi, S. Chiashi, S. Maruyama, Chem. Phys. Lett. 374 (2003) 53.

4. Y. Murakami, S. Chiashi, Y. Miyauchi, M. Hu, M. Ogura, T. Okubo, S. Maruyama, Chem. Phys. Lett., 385, 298-303 (2004).

5. S. Maruyama, Y. Miyauchi, T. Edamura, Y. Igarashi, S. Chiashi, Y. Murakami, Chem. Phys. Lett. 375, 553 (2003).

6. M.J. O’Connell, S.M. Bachilo, C.B. Huffman, V.C. Moore, M.S. Strano, E.H. Haroz, K.L. Rialon, P.J. Boul, W.H. Noon, C. Kittrell, J. Ma, R.H. Hauge, R.B. Weisman, R.E. Smalley, Science 297, 593 (2002).

7. S.M. Bachilo, M.S. Strano, C. Kittrell, R.H. Hauge, R.E. Smalley, R.B. Weisman, Science 298, 2361 (2002).

8. Y. Miyauchi, S. Chiashi, Y. Murakami, Y. Hayashida, S. Maruyama, Chem. Phys. Lett. 387, 198 (2004).

9. S. G. Chou, F. Plentz, J. Jiang, R. Saito, D. Nezich, H. B. Ribeiro, A. Jorio, M. A. Pimenta, Ge. G. Samsonidze, A. P. Santos, M. Zheng, G. B. Onoa, E. D. Semke, G. Dresselhaus, M. S. Dresselhaus, Phys. Rev. Lett. 94, 127402 (2005).

10. V. Perebeinos, J. Tersoff, Ph. Avouris, Phys. Rev. Lett. 94, 027402 (2005).

11. Y. Miyauchi, S. Chiashi, S. Maruyama, Phys. Rev. Lett., to be submitted.

12. Y. Miyauchi, S. Maruyama, Phys. Rev. Lett., to be submitted. 